\title{
Fractionally Sampled Linear Detectors for DS-CDMA
}

\author{
D.R. Brown, D.L. Anair, and C.R. Johnson, Jr.* \\ Cornell University \\ Ithaca, NY 14853
}

\begin{abstract}
In this paper we analyze the performance of fractionally chip sampled linear multi-user detectors for DirectSequence CDMA communication systems. We consider a general DS-CDMA system model accounting for user asynchronism and frequency selective propagation channels. Analysis shows that FIR linear detectors with chip rate sampling cannot perfectly recover $N$ or more users for a system with spreading gain $N$ in the presence of frequency selective channel dynamics or user asynchronism. Drawing inspiration from fractionally spaced equalization, we propose the fractionally chip sampled receiver and show that a FIR linear detector may be able to perfectly recover $\geq N$ users.
\end{abstract}

\section{Introduction}

This paper considers the problem of demodulating digitally modulated signals in the presence of multi-access and multipath interference with a linear multi-user detector. Linear multi-user detectors for DS-CDMA systems have received increased attention due to their advantages of relatively low complexity over the optimal MLSE detector and significantly improved performance over the conventional matched filter detector.

In this paper we investigate the advantages of fractional chip sampling for linear multi-user detectors proposed in [6] where the sampling period of the receiver is some fraction of the chip duration $T_{c}$. Fractionally sampled equalizers (FSEs) for single user linear detection have been studied since the late 1960's and their desirable properties have led to several commercial applications [8]. The documented advantages of FSEs include robustness to timing phase errors [7], the ability to effectively compensate for more severe delay distortion than a baud spaced equalizer [1], and the existence of FIR zero-forcing (ZF) solutions for data distorted by a FIR channel [3]. The necessary and sufficient

\footnotetext{
* Supported in part by NSF grants MIP-9509011, ECS-9528363, and ECS-9811297 and Applied Signal Technology.
}

conditions for the existence of FIR ZF solutions for a (single user) FSE can be summarized as

- sufficient equalizer length,

- subchannel disparity, and

- no additive channel noise.

This paper examines similar conditions for multi-user DSCDMA communication systems. In particular, we focus on deriving a length condition necessary for the existence of ZF solutions which, in the absence of noise, perfectly recover the transmitted symbols by completely canceling all multi-access and multipath interference. The derived length condition indicates that FIR linear detectors with chip rate sampling are unable to perfectly recover $K \geq N$ users in the presence of multipath channel dynamics or user asynchronism. Our necessary length condition also implies that FIR fractionally chip sampled multi-user detectors may be able to perfectly recover transmitted symbols from $K \geq N$ users.

Simulation results verify the analysis and additionally demonstrate that fractionally chip sampled multi-user detectors may also provide significant performance advantages in the presence of additive channel noise.

\section{Discrete Time System Model}

Consider the baseband asynchronous DS-CDMA system model shown in Figure 1. Let $N$ represent the spreading gain common to all users and assume the spreading codes are periodic with period $N$. Let $h^{(k)}(t)$ represent the combined system impulse response of the pulse shaping filter, the $k^{t h}$ user's (fixed or slowly time varying) channel, and the receiver input filter The baseband signal $r(t)$ at the input of the sampler may be written as

$$
\begin{aligned}
r(t)=\sum_{k=1}^{K} \sum_{\ell=-\infty}^{+\infty} u_{\ell / P}^{(k)} h^{(k)}( & \left.t-\ell T_{c} / P-\tau^{(k)}\right) \\
& +\int_{-\infty}^{+\infty} w(\lambda) \phi(t-\lambda) d \lambda
\end{aligned}
$$




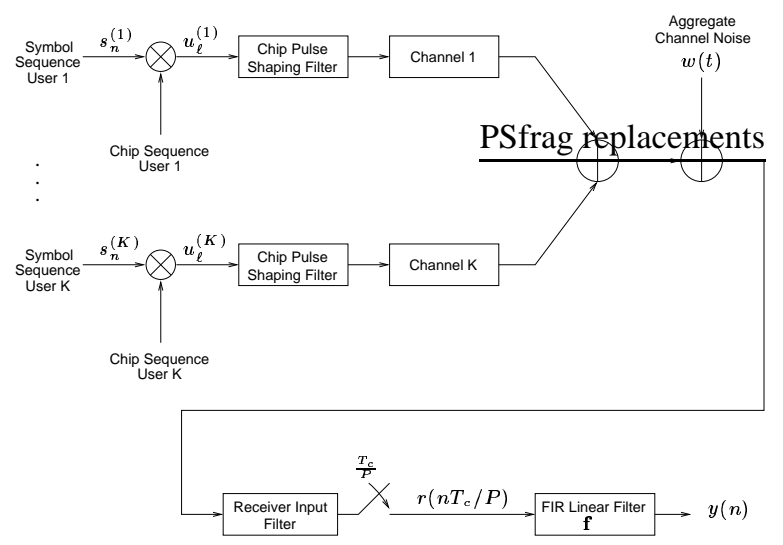

Figure 1. DS-CDMA system model.

where $u_{\ell / P}^{(k)}$ represents the spread (and oversampled) discrete information bearing sequence of the $k^{t h}$ user, $T_{c}$ is the chip duration, $\tau^{(k)} \in\left[0, N T_{c}\right)$ is an arbitrary delay with respect to some reference, $w(t)$ is the aggregate additive channel noise, and $\phi(t)$ is the impulse response of the receiver input filter. Note that $u_{\ell / P}^{(k)}=0$ for $\ell / P \notin \mathbb{Z}$ where $\mathbb{Z}$ denotes the set of all integers. The output of the sampler may then be expressed as

$$
\begin{array}{r}
r\left(n T_{c} / P\right)= \\
\sum_{k=1}^{K} \sum_{\ell=-\infty}^{+\infty} u_{\ell / P}^{(k)} h^{(k)}\left(n T_{c} / P-\ell T_{c} / P-\tau^{(k)}\right) \\
+\tilde{w}\left(n T_{c} / P\right)
\end{array}
$$

where $\tilde{w}\left(n T_{c} / P\right)$ represents the sampled receiver filtered aggregate channel noise.

Denoting the $k^{t h}$ user's symbol delay as $\tau^{(k)}=$ $\left(a^{(k)}+\alpha^{(k)}\right) T_{c} / P$ where $a^{(k)}$ and $\alpha^{(k)}$ represent the integer and fractional number of samples in the delay, respectively, we can rewrite (2) as

$$
\begin{aligned}
& r\left(n T_{c} / P\right)= \\
& \sum_{k=1}^{K} \sum_{\ell=-\infty}^{+\infty} u_{\frac{\ell-a^{(k)}}{P}}^{(k)} h^{(k)}\left((n-\ell) T_{c} / P-\alpha^{(k)} T_{c} / P\right) \\
& +\tilde{w}\left(n T_{c} / P\right) .
\end{aligned}
$$

Assuming that $h^{(k)}(t)$ is FIR with support $\left[0, L T_{c} / P\right)$ for $k \in\{1,2, \ldots, K\}$ we can rewrite (3) as

$$
\begin{array}{r}
r\left(n T_{c} / P\right)= \\
\sum_{k=1}^{K} \sum_{m \in \mathcal{M}^{(k)}} u_{\frac{n-m-a^{(k)}}{P}}^{(k)} h^{(k)}\left(m T_{c} / P-\alpha^{(k)} T_{c} / P\right) \\
+\tilde{w}\left(n T_{c} / P\right)
\end{array}
$$

where

$$
\mathcal{M}^{(k)}=\left\{m: m \in \mathbb{Z} \text { and } m-\alpha^{(k)} \in[0, L)\right\} .
$$

Note that there are exactly $L$ terms in the inner summation and approximately $\frac{P-1}{P}$ of these terms are zero since $u_{p / q}=0$ for $p / q \notin \mathbb{Z}$.

Given a linear detector $\mathbf{f}$ with $N_{f}<\infty$ taps, (4) enables us to directly express the output of the linear detector in terms of finite dimensional linear operators on a vector of source symbols. The linear detector output may be expressed as $y(n)=\mathbf{f}^{\top} \mathbf{r}(n)$ where $\mathbf{r}(n)=$ $\left[r_{n}, r_{n-1}, \ldots, r_{n-N_{f}+1}\right]^{\top}$ and $r_{n}=r\left(n T_{c} / P\right)$. In light of the linear convolution in (4), let $\mathbf{H}^{(k)}$ denote the $N_{f} \times$ $N_{f}+L-1$ dimensional convolution matrix such that

$$
y(n)=\mathbf{f}^{\top}\left[\sum_{k=1}^{K} \mathbf{H}^{(k)} \mathbf{u}^{(k)}(n)+\tilde{\mathbf{w}}(n)\right]
$$

where $\mathbf{u}^{(k)}(n)$ is the $k^{t h}$ user's $a^{(k)}$-sample delayed vector of (oversampled) chip rate information constructed from (4). In general, $\mathbf{H}^{(k)}$ has the Toeplitz form

$$
\mathbf{H}^{(k)}=\left[\begin{array}{cccccc}
h_{0}^{(k)} & h_{1}^{(k)} & \ldots & h_{L-1}^{(k)} & & \\
& \ddots & & & \ddots & \\
& & h_{0}^{(k)} & h_{1}^{(k)} & \ldots & h_{L-1}^{(k)}
\end{array}\right] .
$$

Recognizing that $\mathbf{u}^{(k)}(n)$ may be formed from a linear combination source symbols, we can rewrite (5) as

$$
y(n)=\mathbf{f}^{\top}\left[\sum_{k=1}^{K} \mathbf{H}^{(k)} \mathbf{C}^{(\mathbf{k})} \mathbf{s}^{(k)}(n)+\tilde{\mathbf{w}}(n)\right]
$$

where $\mathbf{C}^{(k)}$ is the map from the $k^{\text {th }}$ user's symbol sequence $\mathbf{s}^{(k)}(n)=\left[s_{n}^{(k)}, s_{n-1}^{(k)}, \ldots, s_{n-Q^{(k)}+1}^{(k)}\right]^{\top}$ to $\mathbf{u}^{(k)}(n)$. In general, $\mathbf{C}^{(k)}$ has the form

$$
\mathbf{C}^{(k)}=\left[\begin{array}{lllll}
\overline{\mathbf{c}^{(k)}} & & & \\
& \mathbf{c}^{(k)} & & \\
& & \ddots & \\
& & \mathbf{c}^{(k)} & \\
& & & \underline{\mathbf{c}^{(k)}}
\end{array}\right] .
$$

where $\mathbf{c}^{(k)}$ is a column vector of length $N P$ representing the $k^{t h}$ user's (oversampled) spreading code. The expressions $\overline{\mathbf{c}^{(k)}}$ and $\underline{\mathbf{c}^{(k)}}$ denote spreading codes which may be upper or lower-truncated, respectively, due to user asynchronism and the finite observation interval. Finally, constructing a user-ordered stacked source vector $\mathbf{s}(n)=$ $\left[\mathbf{s}^{(1) \top}(n), \mathbf{s}^{(1) \top}(n), \ldots, \mathbf{s}^{(K) \top}(n)\right]^{\top}$, we can rewrite (6) as

$$
y(n)=\mathbf{f}^{\top}[\mathbf{H s}(n)+\tilde{\mathbf{w}}(n)]
$$

where $\mathbf{H}=\left[\begin{array}{llll}\mathbf{H}^{(1)} \mathbf{C}^{(1)} & \mathbf{H}^{(2)} \mathbf{C}^{(2)} & \ldots & \mathbf{H}^{(K)} \mathbf{C}^{(K)}\end{array}\right]$. 


\section{Common Linear Multi-user Detectors}

Given the discrete time linear model in (7) it is straightforward to write expressions for zero-forcing (ZF) and minimum mean square error (MMSE) detectors under arbitrary asynchronism and multipath channels. We have intentionally avoided deriving the "decorrelating" detector since it is conventionally defined without consideration for multipath interference. The $\mathrm{ZF}$ detector may be viewed as a generalized decorrelating detector that completely cancels the effects of both multi-user and multipath interference [2].

Given a desired user $k$ and symbol delay $\delta$, a ZF detector is defined as any element of the set $\left\{\mathbf{f}: \mathbf{f}^{\top} \mathbf{r}(n)=\right.$ $\left.s^{(k)}(n-\delta)\right\}$. Let $Q=\sum_{k=1}^{K} Q^{(k)}$ represent the total number of symbols in the observation interval or, equivalently, the number of columns in $\mathbf{H}$. The $\mathrm{ZF}$ detector exists for all $k$ and $\delta$ if and only if the span of the columns of $\mathbf{H}^{\top}$ is all of $\mathbb{R}^{Q}$. Since $\mathbf{H} \in \mathbb{R}^{N_{f} \times Q}$ then this condition is equivalent to requiring that $\operatorname{rank}(\mathbf{H})=Q$ or $\mathbf{H}$ must have full column rank. Under this assumption, the unique minimum norm ZF linear detector may be written as

$$
\mathbf{f}_{Z F}=\left(\mathbf{H}^{\top}\right)^{\dagger} \mathbf{e}_{k, \delta}
$$

where $\mathbf{e}_{k, \delta}$ is a column vector with all elements equal to zero except a value of one in the position corresponding to the $s^{(k)}(n-\delta)$ position in $\mathbf{s}(n)$ and $\dagger$ denotes the MoorePenrose pseudoinverse.

Given a desired user $k$ and symbol delay $\delta$, the MMSE detector is defined as

$$
\mathbf{f}_{M M S E}=\arg \min _{\mathbf{f}} E\left(\left|\mathbf{f}^{\top} \mathbf{r}(n)-s^{(k)}(n-\delta)\right|^{2}\right) .
$$

Assuming that the source symbols are BPSK and i.i.d., (7) leads to a straightforward closed form expression for the MMSE detector:

$$
\mathbf{f}_{M M S E}=\left(\mathbf{H H}^{\top}+\mathbf{R}_{\tilde{w} \tilde{w}}\right)^{\dagger} \mathbf{H} \mathbf{e}_{k, \delta}
$$

where $\mathbf{R}_{\tilde{w} \tilde{w}}$ is the autocorrelation matrix of the receiver filtered noise.

\section{System Matrix Dimensional Analysis}

This section applies the previous development of the discrete time model to analyze the effects of linear detector length $\left(N_{f}\right)$ on the existence of ZF solutions for $\mathbf{f}$. To be specific, we will require that $\mathrm{ZF}$ solutions exist for all $\delta=0,1,2, \ldots, Q^{(k)}-1$ for each $k \in\{1,2, \ldots, K\}$. Since these ZF detectors exist if and only if $\mathbf{H}$ has full column rank, we recognize that a necessary condition for full column rank is that $\mathbf{H}$ must be "tall" in the sense that it must have at least as many rows than columns. We note that the tall condition is not sufficient for full column rank in the same sense that length conditions are not sufficient for the existence of zero-forcing FSEs for single user equalization.

Inspection of the individual users' sub-system matrices $\mathbf{H}^{(k)} \mathbf{C}^{(k)}$ allows us to express the number of columns per user as

$$
Q^{(k)}=\left\lceil\frac{N_{f}+L-1}{N P}\right\rceil+1^{(k)}
$$

where $1^{(k)} \in\{0,1\}$ is a term representing the effects of user asynchronism on $\mathbf{H}$. To be precise, $1^{(k)}=1$ is equivalent to the condition that the $k^{t h}$ user contributes an additional column to the system matrix by having an additional symbol in the observation interval when compared to a synchronized user. As an example, Figure 2 shows a two user asynchronous scenario where $1^{(1)}=1$ and $1^{(2)}=0$. Note that the overlap in the bits is due to multipath channel effects when $L>1$.

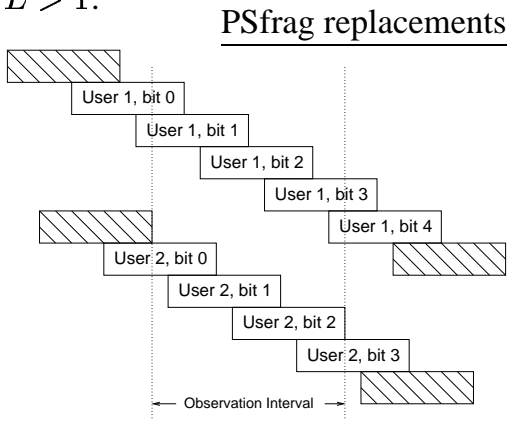

Figure 2. Example of asynchronous users.

In the synchronous case $1^{(k)}=0 \forall k$ and in the asynchronous case $1^{(k)}$ is a function of $\tau^{(k)}, N, P, L$, and $N_{f}$. The exact expression for $1^{(k)}$ is not required to develop the necessary length condition.

It follows directly from (8) that the "tall" condition necessary for full column rank may be stated as

$$
N_{f} \geq K\left\lceil\frac{N_{f}+L-1}{N P}\right\rceil+\sum_{k=1}^{K} 1^{(k)} .
$$

We consider the implications of this result in the following examples.

\section{Examples}

1. Consider the conventional case with no multipath $(L=$ $1)$, chip-rate sampling $(P=1)$, and synchronous users $\left(1^{(k)}=0 \forall k\right)$. If the number of users equals the spreading gain $(K=N)$ then (9) reduces to

$$
N_{f} \geq N\left\lceil\frac{N_{f}}{N}\right\rceil
$$


which is satisfied when $N_{f}=N$ (or any positive integer multiple of $N$ ). This agrees with the well known result for decorrelating detectors [4] where the decorrelating detector is realized as a bank of code matched filters $\mathbf{X}$ followed by the inverse of the $K \times K$ matrix $\mathbf{R}$ of cross-correlations between spreading codes, i.e., $\mathbf{y}(n)=\mathbf{R}^{-1} \mathbf{X r}(n)$. Since $\mathbf{R}^{-1} \mathbf{X}$ has dimensions $K \times N$, it follows that the decorrelating detector is equivalent to an $N$-tap, chip-spaced, linear filter for each user. If the spreading codes are linearly independent then the decorrelating detector achieves perfect symbol recovery and is equivalent to a ZF detector.

2. Consider the previous case with the addition of multipath channels $(L>1)$. When the number of users equals the spreading gain (9) reduces to

$$
N_{f} \geq N\left\lceil\frac{N_{f}+L-1}{N}\right\rceil \geq N_{f}+L-1>N_{f}
$$

which implies that no finite value for $N_{f}$ can cause $\mathbf{H}$ to be tall. The failure to satisfy this necessary condition implies that FIR ZF detectors do not exist for $K=N$ synchronous users in the presence of multipath interference with chip rate sampling.

3. Consider the first case with the addition of user asynchronism such that $1^{(k)}=1$ for at least one user. When the number of users equals the spreading gain (9) reduces to

$$
N_{f} \geq N\left\lceil\frac{N_{f}}{N}\right\rceil+\sum_{k=1}^{K} 1^{(k)} \geq N_{f}+\sum_{k=1}^{K} 1^{(k)}>N_{f}
$$

which implies that no finite value for $N_{f}$ can cause $\mathbf{H}$ to be tall. Again, the failure to satisfy this necessary condition implies that FIR ZF detectors do not exist for $K=N$ asynchronous users with chip rate sampling.

4. Consider the case of an oversampling receiver with $P=2$ and $K=2 N-1$. Note that the number of users is greater than the spreading gain. Assume further that the users are asynchronous. In this case, we can manipulate (9) to write

$$
N_{f} \geq(2 N-1)(L-1+\gamma)+2 N \sum_{k=1}^{K} 1^{(k)}
$$

where $\left\lceil\frac{N_{f}+L-1}{N P}\right]=\frac{N_{f}+L-1}{N P}+\frac{\gamma}{N P}$ and $\gamma \in$ $\{0,1, \ldots, N P-1\}$. Observe that even for worst case asynchronism, $N_{f}$ is finite for $N<\infty$ and $L<\infty$.

The next section verifies these results with simulations and demonstrates that, in addition to the aforementioned desirable properties in a noiseless scenario, the fractionally sampled receiver may provide improved performance in the presence of additive channel noise as well.

\section{Simulations}

Figure 3 shows a comparison between MMSE solutions for $T_{c}$ and $T_{c} / 2$ sampled linear detectors in a noiseless, synchronous communication scenario with multipath interference. To provide a fair comparison, the channel delay spread is fixed at $\left[0,14 T_{c}\right)$ which implies that $L=14$ and $L=28$ in the $T_{c}$ and $T_{c} / 2$ sampled simulations, respectively. The transmit filter is assumed to have square root raised cosine spectrum with excess bandwidth $\beta=0.2$ and the propagation channel coefficients are random. The receiver input filter is an ideal low pass filter with cutoff $\frac{P}{2 T_{c}}$. The length $N=8$ spreading codes are also random (with elements in $\{-1,+1\}$ ). The linear detector length in both cases is $N_{f}=32$ which satisfies the length condition in (9) for the $T_{c} / 2$ sampled receiver.
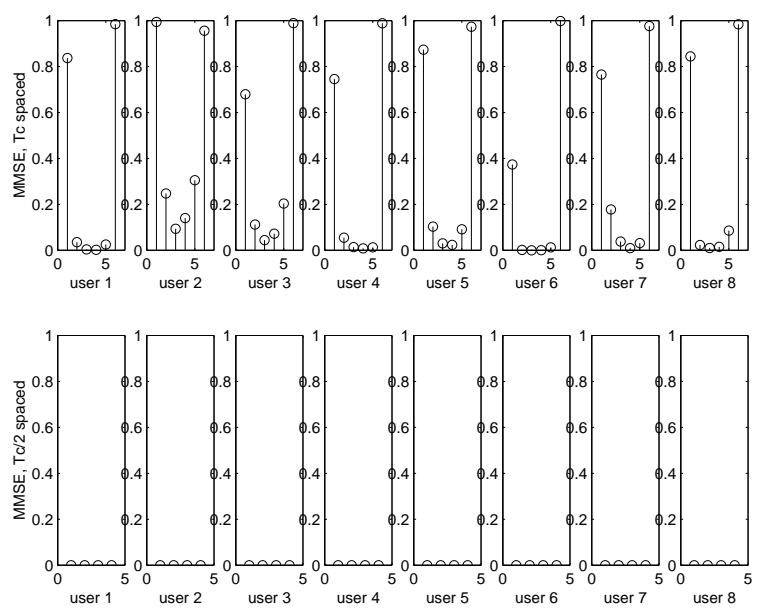

Figure 3. MMSE by user and delay for $T_{c}$ and $T_{c} / 2$ sampled linear detectors. Simulation parameters: channel support $\left[0,14 T_{c}\right)$, spreading gain $N=8$, $K=8$ equal power users, $N_{f}=32$.

Observe that the $T_{c} / 2$ sampled linear detector is able to perfectly recover the symbols of all users at all delays implying that the linear detector completely canceled both multi-user and multipath interference. Inspection of the system matrix confirmed that full column rank was achieved for the fractionally sampled receiver and that $\mathrm{ZF}$ solutions exist for all users at all possible delays. The $T_{c}$ sampled detector exhibits considerably worse performance since the system matrix is not full column rank for any finite choice of $N_{f}$ when $K=N$ and $L>1$.

Note that the MMSE solutions may be indexed by user and delay, i.e., $\mathrm{MMSE}_{k, \delta}=E\left(\left|s^{(k)}(n-\delta)-y(n)\right|^{2}\right)$ where $y(n)$ is the output of the appropriate MMSE linear detector. We will use this notation in the next simulation. 
Figure 4 is a Monte Carlo comparison between $T_{c}$ and $T_{c} / 2$ sampled linear detectors in the presence of AWGN with synchronous and asynchronous equal power users. The asynchronous users each have uniformly distributed delay over $\left[0, N T_{c}\right)$. As in the first simulation, MMSE is calculated for all users at all possible delays but in this case the delay-optimal MMSE, given by $\mathrm{MMSE}_{k^{*}}=$ $\min _{\delta} \mathrm{MMSE}_{k, \delta}$, is selected for each user and then averaged over all users and 500 experiments. The noise variance $\sigma_{w}^{2}=0.1$. The linear detector length $N_{f}$ is varied from 2 to 100. All other parameters are as in the first simulation.
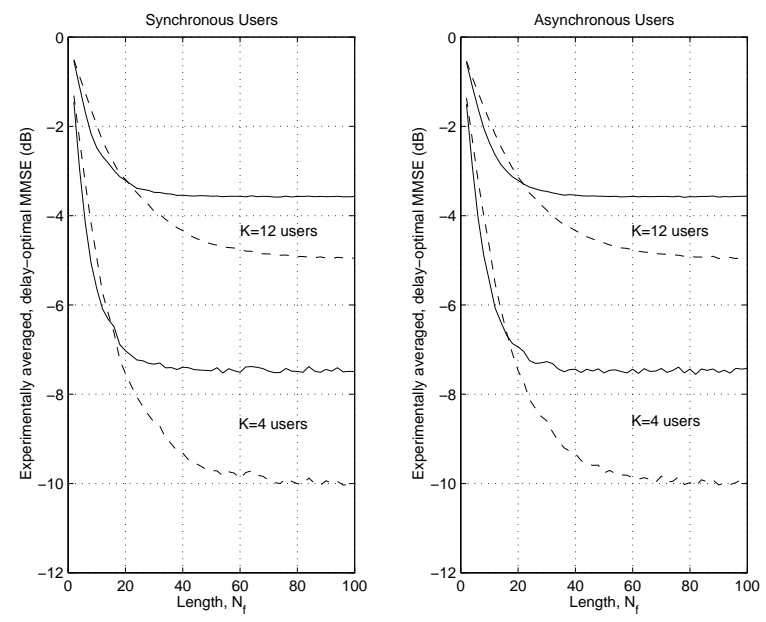

Figure 4. Averaged delay-optimal MMSE for $T_{c}$ (solid lines) and $T_{c} / 2$ sampled (dashed lines) linear detectors with synchronous and asynchronous equal power users. Simulation parameters: channel support $\left[0,14 T_{c}\right)$, spreading gain $N=8$.

This simulation provides evidence indicating that $T_{c} / 2$ sampled receivers may achieve better MMSE performance than $T_{c}$ sampled receivers in the presence of additive channel noise, but only for larger values of $N_{f}$. The relatively poor performance of the fractionally sampled receiver for small values of $N_{f}$ is due to the fact that, for a fixed value of $N_{f}$, the observation interval of the $T_{c} / 2$ sampled receiver is half that of the $T_{c}$ sampled receiver. In this simulation, values of $N_{f}<42$ cause the $T_{c} / 2$ sampled receiver's observation interval to not contain even one full bit (including multipath) from a synchronous user. Also note that this simulation suggests that the effects of asynchronism on averaged delay-optimal MMSE performance are almost negligible for both $T_{c}$ and $T_{c} / 2$ sampled receivers.

\section{Conclusions}

In this paper we have explored the concept of fractional sampling to improve the performance of linear multiuser detectors for DS-CDMA communication systems. The analysis has shown that fractionally sampled receivers of sufficient length satisfy a necessary condition for the existence of zero-forcing solutions under conditions where no FIR solution exists for chip rate sampled receivers. Furthermore, simulations in the presence of additive channel noise indicate that fractionally sampled receivers may also offer improved MMSE performance over chip rate receivers.

The "robustness to timing phase errors property" of single user FSEs was not explored in this paper for fractionally sampled multi-user detectors and remains an open research topic. Necessary and sufficient conditions for the existence of FIR zero-forcing solutions in DS-CDMA communication systems with arbitrary asynchronism and multipath channels are also a topic of current research.

\section{References}

[1] R.D. Gitlin and S.B. Weinstein, "Fractionally-spaced equalisation: An improved digital transversal equaliser,"Bell System Technical Journal, vol. 60, pp. 275-296, Feb. 1981.

[2] S. Gollamudi, et al, "On the dimensional limitations of linear multiuser detection," in Proc. Thirty-Fifth Annual Allerton Conference on Communication, Control, and Computing (Monticello, IL), pp. tbd, Sep. 25-29, 1998.

[3] C.R. Johnson Jr., et al, "Blind equalization using the constant modulus criterion: A review,"Proceedings of the IEEE, vol. 86, no. 10, pp. 1927-50, Oct. 1998.

[4] R. Lupas and S. Verdu, "Linear multi-user detectors for synchronous code-division multiple-access channels,'IEEE Trans. on Information Theory, vol. 35, pp. 123-136, Jan. 1989.

[5] U. Madhow and M. L. Honig, "MMSE interference suppression for direct-sequence spread-spectrum CDMA,'IEEE Trans. on Communications, vol. 42 , no. 12 , pp. $3178-88$, Dec. 1994.

[6] U. Madhow, "Signal processing for interference suppression in direct-sequence CDMA systems," in Proc. International Conference on Acoustics, Speech and Signal Processing (Atlanta, GA), pp. 1065-8, May 1996.

[7] M. Shafi and D.J. Moore, "Further results on adaptive equalizer improvements for 16 QAM and 64 QAM digital radio,"IEEE Trans. on Communications, vol. 34, no. 1, pp. 5966, Jan. 1986.

[8] J.R. Treichler, I. Fijalkow, and C.R. Johnson, Jr., "Fractionally spaced equalizers: How long should they really be?,"Signal Processing Magazine, vol. 13, pp. 65-81, May 1996. 